\section{Review Article}

\section{Check for updates}

\title{
Medico-Legal Issues of Intraoperative Neuromonitoring in Thyroid Surgery
}

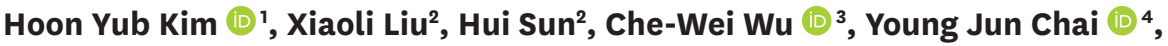 \\ Woong Youn Chung ${ }^{5}$, Ralph Tufano $\mathbb{1}^{6}$, Henning Dralle $\mathbb{1}^{7}$, Matteo Lavazza ${ }^{8}{ }^{8}$, \\ Gianlorenzo Dionigi ${ }^{8}{ }^{8}$
}

'Korea University Medical Center (KUMC) Thyroid Center, Korea University Anam Hospital, Korea University College of Medicine, Seoul, Korea

${ }^{2}$ Division of Thyroid Surgery, Jilin Provincial Key Laboratory of Surgical Translational Medicine, China-Japan Union Hospital of Jilin University, Changchun, China

${ }^{3}$ Department of Otolaryngology, Kaohsiung Medical University Hospital, Kaohsiung Medical University, Kaohsiung, Taiwan

${ }^{4}$ Department of Surgery, Seoul Metropolitan Government-Seoul National University Boramae Medical Center, Seoul, Korea

${ }^{5}$ Department of Surgery, Yonsei University College of Medicine, Seoul, Korea

${ }^{6}$ Department of Otolaryngology-Head and Neck Surgery, The Johns Hopkins University School of Medicine, Baltimore, MD, USA

${ }^{7}$ Endocrine Surgery, Universitätsklinikum Essen AöR Medizinisches Zentrum, Essen, Germany

${ }^{8} 1$ st Division of General Surgery, Research Center for Endocrine Surgery, Department of Medicine and Surgery, ASST dei Sette Laghi Ospedale di Circolo e Fondazione Macchi, Polo Universitario, University of Insubria (Varese-Como), Varese, Italy

\section{ABSTRACT}

Advances in intraoperative neuromonitoring (IONM) in thyroid surgery have provided significant insights into recurrent laryngeal nerve function during thyroid surgery. Despite the limitations and necessary caution when using intraoperative monitors to interpret neural function, these technologies have been definite steps in the right direction for assessing neural integrity and safe surgical strategy during thyroid operations. The techniques discussed minimize the adverse sequelae of a variety of thyroid gland procedures, reducing the morbidity rates/risks in the perioperative period. Furthermore, it is likely that such monitoring will become a standard of care. Accurate, reliable and continuous monitoring is essential, and on-going large studies with definable end points will be necessary. The use of monitoring, such as continuous one, may improve cost efficiency by reducing permanent nerve injuries. A danger in this process, however, is the potential for public opinion, outside regulatory bodies, or medico-legal implications to drive change and enforce standards of care before appropriate data are available.

Keywords: Thyroid gland; Recurrent laryngeal nerve; Malpractice; Recurrent laryngeal nerve injuries; Thyroidectomy

\section{INTRODUCTION}

Little information is available regarding the frequency of thyroidectomy-related malpractice claims. Few studies have assessed claims that were settled or dropped before trial, providing only a limited view of the medico-legal environment. From the database of the Physician Insurers Association of America (PIAA), claims from 1985 to 2008 with thyroid-related 
Henning Dralle (D)

https://orcid.org/0000-0003-4710-8193

Matteo Lavazza (D)

https://orcid.org/0000-0001-6467-3800

Gianlorenzo Dionigi (iD

https://orcid.org/0000-0003-0864-6087

\section{Author Contributions}

Conceptualization: Hoon Yub Kim, Xiaoli

Liu, Hui Sun, Che-Wei Wu, Young Jun Chai,

Woong Youn Chung, Ralph Tufano, Henning

Dralle, Matteo Lavazza, Gianlorenzo Dionigi;

Data curation: Gianlorenzo Dionigi; Formal

analysis: Matteo Lavazza; Funding acquisition:

not required; Investigation: Hoon Yub Kim;

Methodology: Matteo Lavazza; Project

administration: Hoon Yub Kim, Young Jun

Chai; Resources: Matteo Lavazza; Software:

Matteo Lavazza; Supervision: Hoon Yub Kim,

Xiaoli Liu, Hui Sun, Che-Wei Wu, Young Jun

Chai, Woong Youn Chung, Ralph Tufano,

Henning Dralle, Matteo Lavazza, Gianlorenzo

Dionigi; Validation: Hoon Yub Kim, Young

Jun Chai; Visualization: Gianlorenzo Dionigi;

Writing - original draft: Gianlorenzo Dionigi;

Writing - review \& editing: Young Jun Chai.

Conflict of Interest

No potential conflict of interest relevant to this article was reported. procedure codes were evaluated for claimant information, insured's specialty, loss description, causation, and claim outcomes (1). During the 24-year period reviewed, 380 claims related to thyroid surgery were reported. One hundred twenty-eight claims (33.7\% of total claims) resulted in an indemnity payment either due to settlement or a finding against the defendant. The average indemnity payment was $\$ 185,366$ (range, $\$ 363$ to $\$ 2,000,000$ ). Among the cases in which a specific outcome was reported, 55 were related to laryngeal nerve injury or voice disturbance. No substantial change occurred in the incidence of claims across the study period. During this time, approximately $\$ 2,585,000$ thyroidectomies were performed. Extrapolating from the PIAA data, this represents an estimated 5.9 claims per 10,000 cases (1).

Using the LexisNexis Academic legal database, a retrospective review of all relevant federal and state cases from 1989 to 2009 was performed using the search terms "thyroid," "surgery," and "medical malpractice" (2). A total of 143 medical malpractice cases involving thyroid surgery were retrieved from our search from 1989 to 2009. After reviewing all cases, 33 cases in which the alleged negligence occurred after thyroid surgery were used for analysis. Of these cases, 15 involved recurrent laryngeal nerve (RLN) injury. Interestingly, no mention of RLN monitoring was noted in any of the cases (2).

For the evaluation of a vocal cord paralysis (VCP) that has occurred after a thyroid surgery using intraoperative neuromonitoring (IONM), it is of crucial importance to take into account whether or not the published standards for proper use of IONM were applied correctly, and whether or not changes of neuromonitoring signal and electromyogram (EMG) were correctly interpreted intraoperatively and accordingly responded in a risk-oriented manner with regard to the further operational strategy of the second side thyroid lobe $(3,4)$.

On the basis of results from the current literature, it can be concluded that the frequency of VCP has decreased over the years since the introduction of IONM in thyroid operations (5). However, in individual cases, bilateral paresis can be reliably avoided, but unilateral paresis cannot be safely avoided with the use of the $\operatorname{IONM}(6,7)$.

The current report derives from a review of the literature and personal experience on malpractice claims. Since no systematic analysis has been performed so far for the assessment of postoperative paresis of the superior laryngeal nerve (SLN) and continuous intraoperative neuromonitoring (CIONM), the present study is limited to the assessment of postoperative VCP as a result of injury to the RLN and of intermitted IONM.

Briefly, the IONM legal issues that will be presented here can be summarized into: 1) bilateral RLN palsy prevention by IONM; 2) EMG documentation from monitoring; 3) the use of IONM in difficult cases; 4) standard of care of monitoring in thyroid surgery; 5) appropriate and standardized use of IONM; and 6) costs of IONM and RLN palsy (Table 1).

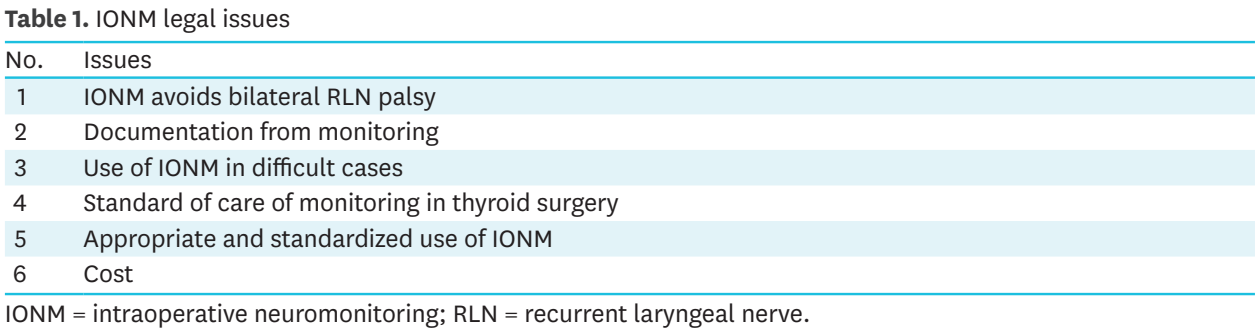




\section{RISK FACTORS FOR INJURIES OF THE RLN}

RLN injury is a well-known and serious complication of thyroid surgery. A recent study estimated the frequency of post-thyroidectomy RLN palsy and identified possible risk factors (8). Based on the Danish national thyroid surgery database, 6,859 patients treated with thyroid surgery from January 1, 2001 to December 31, 2008, at the Danish Departments of Otolaryngology-Head and Neck Surgery (ENT-HNS) were analyzed (8). Unilateral RLN palsy was found in $2.1 \%$, while $0.1 \%$ had bilateral RLN palsy. In benign histology, RLN palsies were registered in $1.3 \%$. Malignant histology and accordingly neck dissection were the most predominant risk factors with a relative risk (RR) of 5.4 and 5.8, respectively (8). In benign cases, previously performed thyroid surgery had an RR of 10.4. Malignant histology, neck dissection, and previous thyroid surgery are the strongest predictors for RLN palsy (8).

The surgical experience pertaining to the identification, exposure, and protection of the RLN has a demonstrable influence on the nerve paresis rates $(8,9)$. A study showed that the rate of postoperative permanent laryngeal nerve paresis correlated directly with the number of nerves at risk (NAR). A palsy rate of less than $1 \%$ was achieved when the operating frequency was 60 and more NAR per surgeon and year (9).

An increased risk of RLN palsy is preoperatively detectable when a locally advanced cancer is diagnosed or a retrosternal huge struma is detected on imaging. Graves' disease, Hashimoto, de Quervain, or Riedel's thyroiditis also indicate a potentially increased risk of RLN palsy $(8,9)$. Particularly high is the risk of bilateral paresis with pre-existing unilateral paresis and the need for bilateral resection. In this situation, it is critical to check whether there is a need for a resection to be performed contralaterally to the pre-existing RLN paretic side.

Anatomic criteria determining patients with high-risk thyroid surgery have been described and defined based mostly on expert opinion. Preoperatively unpredictable risk situations, which can only be detected intraoperatively, are one of the main reasons for using neuromonitoring not only selectively but also routinely. Examples of intraoperative risk situations include: 1) atypical RLN pattern anterior to the thyroid gland; 2) RLN anterior to the Zuckerkandl's tuberculum nodule; 3) fixed, splayed, or entrapped RLN with capsular association through fascial bands; 4) vessels or goiters change; 5) invaded RLN; 6) posterior ligament of Berry nerve; 7) thin RLN (<1 $\mathrm{mm})$; 8) ramificated or antevascular RLN; or 9) non-RLN occurring in about $1.5 \%$ of the thyroid gland operations (Table 2). Atypical courses of the RLN are observed in approximately a quarter of the thyroid gland operations. Therefore, IONM-based treatments are recommended and the centralization of thyroid surgery in larger units using IONM might improve quality (8).

\begin{tabular}{ll}
\multicolumn{2}{l}{ Table 2. } \\
\hline No. & Situations \\
\hline 1 & Atypical RLN pattern \\
2 & RLN anterior to the thyroid gland \\
3 & RLN fixed, splayed or entrapped \\
4 & RLN posterior to Berry ligament \\
5 & Anteriorly located RLN to the Zuckerkandl's tuberculum (posterior nodule) \\
6 & Branched RLN \\
7 & Antevascular RLN \\
8 & Thin RLN \\
9 & Invaded RLN \\
10 & Non-RLN \\
\hline
\end{tabular}

$\mathrm{RLN}=$ recurrent laryngeal nerve. 


\section{INTERPRETATION OF NEUROMONITORING EMG SIGNALS}

The correct reading and interpretation of neuromonitoring EMG signals are essential to avoid any medico-legal errors.

\section{Preoperative laryngoscopy (L1)}

The standards of RLN management include extensive knowledge of RLN anatomy, visual identification and functional confirmation of RLN, nerve exposure, training and certainly pre- and postoperative laryngeal examination (L1 and L2, respectively). Both L1 and L2 are essential in all cases, while IONM is also essential in the development phase to improve the prognostic correlation between neural stimulation and pre- and postoperative glottis function (3). L1 is a reference for IONM. Since a normal movement/action potential of the vocal cords (VCs) is recorded in approximately 10\% of the cases even in RLN injuries, an interpretation of the IONM signal and EMG is not possible without an understanding of the L1 findings of the VCs (10). Therefore, IONM is not suitable for intraoperative decisionmaking in the case of pre-existing vocal paresis, regardless of whether symptomatic or asymptomatic, since, even with ipsilateral normal EMG action potential after a contralateral RLN paresis, postoperative bilateral paresis may occur (10).

\section{Pre-dissection vagal nerve (VN) stimulation (V1)}

$\mathrm{V} 1$ is the reference for RLN identification, confirmation, stimulation, and dissection (Fig. 1). Moreover, before starting the preparative steps for the exposure and resection of the thyroid gland, the ipsilateral $\mathrm{VN}$ is stimulated in order to test the functional reliability of the monitoring device and to determine the initial amplitude. An unequivocal definition of normative V1 means that values have been analyzed (11). The $\mathrm{V} 1$ amplitude should be at least $300 \mu \mathrm{V}$, or optimally ${ }^{3} 500 \mu \mathrm{V}(3,11)$. Only for amplitudes exceeding $300 \mu \mathrm{V}$ could an amplitude decrease of more than $50 \%$ of the relevant initial value for the operative procedure be reliably diagnosed. For initial amplitudes below 300-500 $\mu \mathrm{V}$, the position of the EMG tube electrodes should be optimized until an amplitude of at least $300-500 \mu \mathrm{V}$ is displayed in the monitor as the baseline (3).

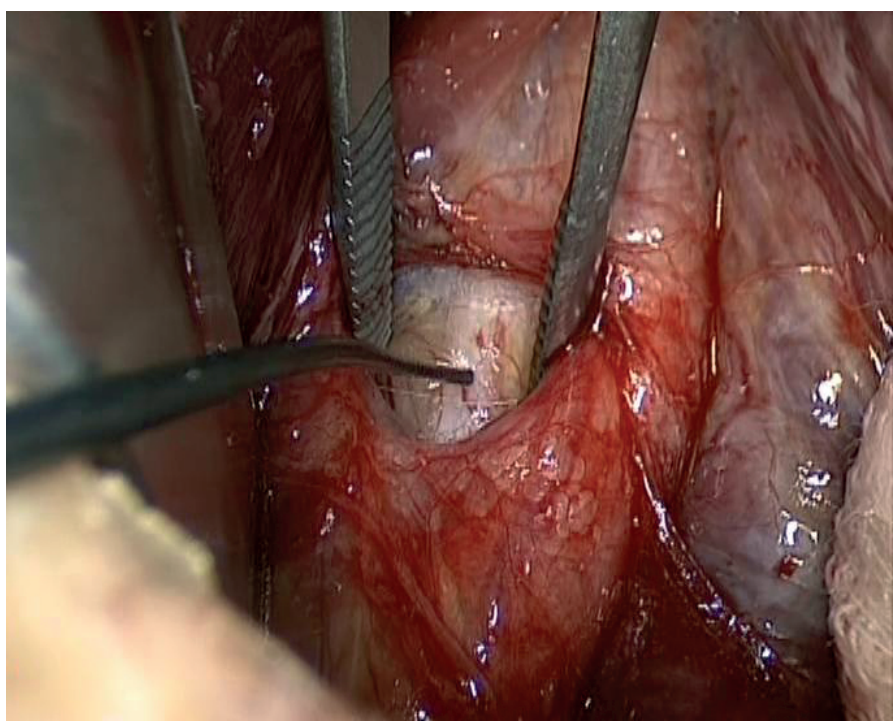

Fig. 1. Pre-dissection VN stimulation (V1). $\mathrm{VN}=$ vagal nerve. 
In fact, high IONM quality is fundamental to make any surgical deliberation, both for safety and to reduce false results. A V1 amplitude of at least $300 \mu \mathrm{V}$ or optimally ${ }^{3} 500 \mu \mathrm{V}$ is the prerequisite for: 1) the diagnosis, interpretation, and verification of a functionally intact RLN; 2) any reduction of EMG signal; 3) "re-entry"/recovery of EMG signals; 4) loss of signals (LOSs); and 5) preoperative VCP (nerve conduction and EMG signal preserved).

A further reason for the initial VN stimulation, especially on the right side, is the early detection of a right non-RLN (12). The existence of this atypical nerve can be secured by VN stimulation, which is initially caudal (i.e., no vagal stimulation signal), then cranial (i.e., vagal stimulation signal above the origin of the non-RLN) (12-14).

\section{Pre-dissection recurrent stimulation (R1)}

R1 should also be performed prior to the exposure and resection of the thyroid gland. It is the first step for the nerve identification and confirmation, and it is intended to recognize the atypical nerve pathway as early as possible (Table 2$)(13,14)$.

\section{Post-resectional vagal nerve stimulation (V2), recurrent stimulation (R2), and postoperative laryngoscopy (L2)}

Irrespective of the operative strategy of a 1-way or 2-way approach, the ipsilateral RLN (R2) and vagal stimulation (V2) after the completion of all operative measures serve for the detection of the RLN function and the prediction of a postoperatively intact or injured vocal fold function (3,13-21). In post-resection intact VN signal and EMG, intact VC mobility can be assumed in more than $99 \%$ of the cases (Table 3). In the case of intraoperative signal loss, an early postoperative VC paresis is present in more than $70 \%$ of the cases, depending on the type of signal loss (type 1, segmental nerve injury; type 2, global nerve injury) (Fig. 2). The rate of non-structural, non-morphological, and non-visible RLN lesions (i.e., nerve traction) is more than $90 \%$ (Table 3 ). Therefore, the predictive value of intraoperative vagal stimulation is low in the case of intraoperative signal loss with regard to long-term vocal function. In cases of structural, visible, and morphological nerve injury (i.e., nerve transection), permanent vocal palsy must always be considered (13-21).

\section{RLN and vagal stimulation during thyroid resection}

The repeated RLN and vagal stimulation during intermittent and continuous neuromonitoring serves for the anatomical nerve course confirmation and simultaneous functional control of the RLN. In that way, before every step (tissue and/or vascular dissection), it is possible to identify the RLN and preserve its anatomical and functional integrity as best as possible. Neuromonitoring, however, does not guarantee a complete safety with regard to the preservation of the nerve function. Indeed, even in the case of an

Table 3. Predictive value of IONM

\begin{tabular}{|c|c|c|c|c|c|c|c|}
\hline Studies & NAR (No.) & Specificity (\%) & Sensitivity (\%) & PPV (\%) & NPV (\%) & EPOVP & PVP \\
\hline Barczyński et al. (5) & 1,000 & 63.0 & 97.1 & 37.8 & 98.9 & $27(2.7)$ & $8(0.8)$ \\
\hline Thomusch et al. (16) & 12,486 & 33.0 & 98.3 & 36.7 & 97.9 & $413(2.7)$ & $104(0.7)$ \\
\hline Beldi et al. (17) & 429 & 40.0 & 98.0 & 67.0 & 91.0 & 37 (8.7) & $6(1.4)$ \\
\hline Hermann et al. (18) & 475 & 57.1 & 99.3 & 87.0 & 96.6 & $43(8.9)$ & $15(3.1)$ \\
\hline Chan et al. (19) & 271 & 53.0 & 94.0 & 35.0 & 97.0 & $15(5.5)$ & $2(0.7)$ \\
\hline Tomoda et al. (4) & 2,197 & 69.3 & 99.7 & 92.1 & 98.5 & $80(3.6)$ & $21(1.0)$ \\
\hline Melin et al. (21) & 3,426 & 85.4 & 99.0 & 68.0 & 99.6 & $82(2.4)$ & NA \\
\hline
\end{tabular}

Values shown are number (\%) not otherwise specified.

IONM = intraoperative neuromonitoring; NAR = nerves at risk; PPV = positive predictive value; NPV = negative predictive value; EPOVP = early postoperative vocal palsy; PVP = permanent vocal palsy; NA = not available. 

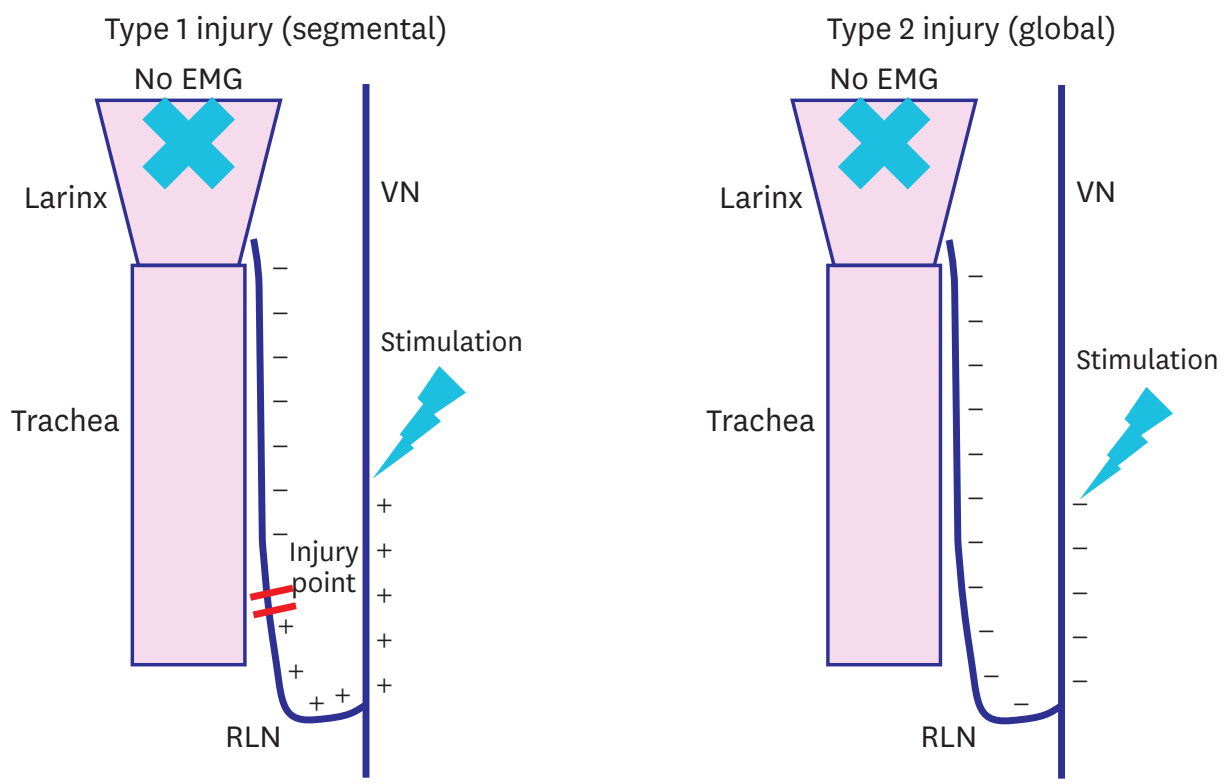

Fig. 2. Type 1 and type 2 injuries.

$E M G$ = electromyogram; $R L N=$ recurrent laryngeal nerve; $V N=$ vagal nerve.

anatomically and structurally preserved nerve, there may be a point or global nerve stress, for example due to nerve tension during thyroid lobe mobilization (13).

\section{Intraoperative signal failure with or without restoration of the RLN function} According to the agreement of the International Neural Monitoring Study Group (INMSG), a signal failure is defined as: 1) a signal loss of the ipsilateral VN;2) no EMG response from initial satisfactory EMG (V1 $>300 \mu \mathrm{V}$ or optimally $\geq 500 \mu \mathrm{V}$ ); and 3) an amplitude drop below $100 \mu \mathrm{V}$ with stimulation at 1-2 $\mathrm{mA}$, with: 1 ) preoperative laryngoscopically intact VC mobility; 2) no laryngeal twitch (LT); and 3) a troubleshooting algorithm applied systematically (3).

Recent investigations have shown that the amplitude of $100 \mu \mathrm{V}$ indicated as a cut-off between intact and injured nerve function as the absolute amplitude of $100 \mu \mathrm{V}$ rather than the relative decrease of the amplitude by less or more than $50 \%$ relative to the initial amplitude for the prediction of the postoperative vocal function is of particular importance (22). The integrity of the VC function can be proven with great certainty if the amplitude recovers more than $50 \%$ of the initial amplitude during the operation (22). On the other hand, if the amplitude is below $50 \%$, the probability of postoperatively intact vocal motility is only $60 \%-80 \%$ (22). For example, for an initial amplitude of $1,000 \mu \mathrm{V}$ and a LOS with subsequent re-amplification of the amplitude to only $300 \mu \mathrm{V}$, a probability of $20 \%-40 \%$ must be expected for postoperatively altered VC mobility. The initial (i.e., maximum) amplitude ("baseline"), optimized at the beginning of the thyroid operation on the basis of the tube position, is thus of enormous importance for the intraoperative prediction of postoperative vocal function (22).

\section{IONM DEVICE TECHNOLOGY AND SAFETY}

\section{Monitoring device technology}

The IONM device technology is based on the principle of combined audio and graphic (amplitude and latency) detection of muscle action potentials of the VC (3). The stimulation 
tone generated by the neuromonitoring device alone, i.e., without simultaneous control of the EMG, is not suitable for distinguishing accurately between an artifact tone and a regular muscle tone. Only simultaneous audio and EMG graphics can provide evidence of the amplitude and latency between stimulation and stimulus response indicating that a regular stimulus response of the ipsilateral vocal muscle is present. In the absence of EMG amplitude and latency, the perceived sound is an artifact. Thus, IONM equipment based on audio and graphic information should be preferred owing to permitting comprehensive documentation, EMG quantification, storage and recording, differentiation between signal and artifacts, research, justification of intraoperative surgical deliberations, and for forensic use (Table 4).

In the case of a stimulation with an EMG amplitude of less than $100 \mu \mathrm{V}$, more than $70 \%$ postoperatively disturbed VC mobility (correct-positive findings) is to be expected. At a stimulation amplitude above $100 \mu \mathrm{V}$, however, a postoperatively intact VC mobility (correct-negative finding) is to be expected in more than $90 \%$ of the cases (Table 3). The false-negative findings (muscle response signal and EMG above $100 \mu \mathrm{V}$ without signal loss, but with postoperative vocal palsy), which are observed in very few cases $(<1 \%)$, are based on the fact that, in the limiting range of the device-determined $100 \mu \mathrm{V}$ in cases with an amplitude drop of more than 50\%, high initial amplitude results in an electrophysiologically positive stimulus response; however, this is not sufficient to produce laryngoscopically intact vocal motility. In contrast, a postoperative recovery of the vocal fold function up to the time of L2 can occur during an intraoperative signal failure with amplitude values below $100 \mu \mathrm{V}$, and thus false-positive findings (about $5 \%$ for LOS type 1 and about $30 \%$ for LOS type 2 ) of IONM.

From a medical perspective, the following aspects of the device technology must be considered:

1. The patient, who has been informed and promised preoperatively of the use of neuromonitoring for his/her thyroid gland operation, assumes that the technique is available and functioning. The surgeon must ensure that the device technology is intact at the time of operation and is used according to the application standards (3).

2. Because IONM is not only a complex technology that can unexpectedly fail at different stages in the period of stimulation but also a technique in which the surgeon can rely on the competent cooperation with the technical personnel and the anesthesiologist (tube positioning), the technology cannot be assessed as a so-called fully manageable risk in the event of an unpredictable failure of the device technology (3).

3. The patient should be informed preoperatively of the possibility of IONM failure.

\begin{tabular}{cl} 
Table & 4. Advantages of IONM equipment based with audio and graphic information \\
\hline No. & Advantages \\
\hline 1 & Comprehensive documentation \\
2 & EMG quantification (amplitude and latency) \\
3 & Storage and recording \\
4 & Differentiation between signal and artifacts \\
5 & Research \\
6 & To justify intraopeative surgical deliberations \\
7 & Forensic use \\
\hline
\end{tabular}

IONM = intraoperative neuromonitoring; EMG = electromyogram. 


\section{IONM safety}

With regard to intraoperative vagal and RLN monitoring, there are no contra-indications for both the intermittent and continuous stimulation. There are also no potentially relevant neurophysiological side effects, i.e., heart, immune modulation, respiration, and gastrointestinal function (23). The VN preparation, like that of the RLN, should be performed gently, and injury to the nerve vessels should be avoided (3).

\section{IONM DOCUMENTATION}

Patient thyroid history, preoperative radiologic imaging, pre-operative symptoms, pre- and postoperative laryngeal examination, exhaustive surgical report with intraoperative findings, identification of RLN, pictures, and videos are important to document any pre-existing airway impairment or correct surgical deliberations.

The documentation of the EMG muscle action potential is possible with all modern IONM devices. IONM converts muscle activity into recorded electromyographical signals, which are often possible to print out. It not only serves the patient's legitimate interest in understanding the use of neuromonitoring but also primarily communicates to the surgeon the important information during the operation (i.e., artifact signal, amplitude decline, and latency increase). Documentation of the final normal neurophysiologic signals of the RLNs at the end of the surgical procedure may have a forensic function, with early differentiation between RLN related and unrelated voice changes. From a medico-legal point of view, it is recommended to record and document the muscle action potential at the beginning (V1; R1) and at the end (V2; R2) of the resection per each side of resection. When IONM is used, its documentation is required and includes time-traceable measures of amplitude, latency, waveform morphology, and magnitude of stimulating current. EMG curves are a proof of intact nerve function. Monitoring nerves during surgical procedures may potentially reduce the medico-legal liability for surgeons, with a reduction of economic loss for the patient, health system, and insurance companies. Finally, the recorded nerve signal is important for early differentiation between RLN related and unrelated voice changes as well (24).

\section{PROCEDURE FOR EMG SIGNAL FAILURE AND VCP}

\section{Troubleshooting algorithm}

Given the potential impact that LOS may have on the surgical plan (i.e., aborting the second side surgery), it is critical that the surgeon who uses IONM is perfectly familiar with the LOS troubleshooting algorithm. With correct application of detailed LOS troubleshooting algorithm, the rate of negative signal at the end of the surgery becomes reduced and the incidence of postoperative VCP in this group becomes substantially higher. The International IONM Study Group recommends adding either LT or contralateral VN stimulation assessments to the noted EMG data. If the noted EMG data is present and there is no LT, but contralateral VN response with stimulation, then the surgeon must consider this to be true LOS and that nerve injury has occurred. Fig. 3 summarizes the troubleshooting algorithm for IONM in case of EMG LOS.

For each failure of the VN signal, it is the first necessary to check whether there is a failure in the IONM device (i.e., dislocation of the electrodes, EMG tube displacement), or if 


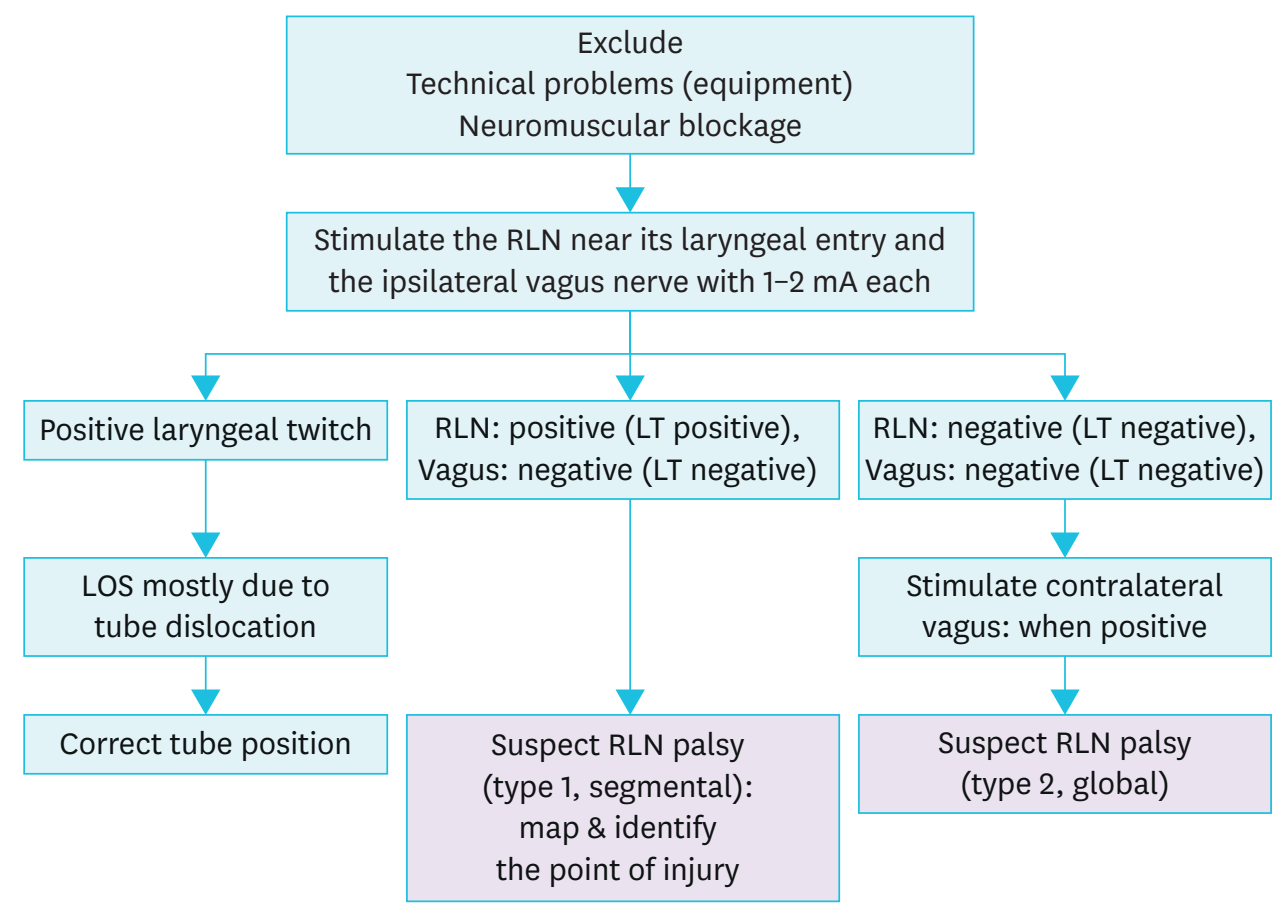

Fig. 3. Troubleshooting algorithm in case of loss of EMG signal. $E M G$ = electromyogram; RLN = recurrent laryngeal nerve; $L O S=$ loss of signal; $L T$ = laryngeal twitch.

anesthesiological muscle-relaxing substances have been administered (3). In the absence of these causes, the loss of the vagal signal indicates a LOS type 1 (segmental lesion, type 1) in the case of an EMG muscular action of the RLN at entry point, or a global loss of the conduction of the stimulus in the absence of muscle action potential of the VN and the RLN (type 2) (Figs. 2 and $\mathbf{3}$ ). The pathogenesis of type 2 signal loss has not yet been clarified; however, both LOS types are likely to result in postoperative VC paresis (approximately 95\% in type $1,70 \%$ in type 2) (13). In case of doubt, the contralateral and functionally intact VN should be stimulated for the purpose of differential diagnosis between a true signal loss or IONM device failure (3).

\section{Surgical consequences of signal loss}

In the case of unilateral thyroid gland resection or in the event of a signal failure after resection of the second side, no further surgical measures are taken if no further interventions, for example a lymphadenectomy, are planned. The side of the signal failure should be resected completely in order to rule out the possibility of an operation on this side in the future.

In the case of the occurrence of bilateral resection and signal failure on the first operated side, the risk of bilateral paresis should be considered (7). In rare cases, such as benign struma, a partial resection, contralateral at a safe distance from the RLN, can be considered. In the case of low-risk carcinomas, however, this should not be done, since the completion necessary to enable radioiodine therapy is associated with an increased RLN risk. Only in the case of differentiated thyroid carcinomas with high risk and the need for an immediate radioiodine therapy, can the contralateral resection be justified despite the very probable paresis on the first side. However, all further measures to increase RLN risk should be avoided, including prophylactic lymph node dissection. 


\section{Operative procedure for pre-existing VCP}

A resection of the contralateral side of a pre-existing VCP should be performed only if there is evidence of: 1) contralateral malignancy from the fine needle aspiration cytology; 2) carcinoma in the dominant (contralateral) side of the thyroid gland; and/or 3) non-operative treatment alternatives from the preoperative interdisciplinary therapy recommendation. If these conditions are not present, and if the intervention is a redo surgery, not only total resection but also a partial resection of any kind (intra- or extracapsular) should be avoided. Indeed, especially in redo interventions, the risk of an unintentional nerve lesion due to scarred changes is not calculable. The course of the nerve is usually altered during reoperative procedures from preoperative surgery, and the nerve is almost permanently drawn to the posterior thyroid gland capsule.

With regard to the use of IONM on the VCP side, it should be borne in mind that a positive vagal EMG, which is observed in approximately $10 \%$ of the cases, should not lead to the contralateral side without a very urgent indication (10).

\section{AVOID BILATERAL VCP}

Reduction of major injury to patients such as bilateral RLN palsy is a great advantage in the modern era of thyroid surgery. A possible consequence of LOS of RLN at the first side is an accurate consideration of optimal contralateral surgery timing. With LOS, the surgeon must consider that the ipsilateral nerve is injured at least temporarily and can subsequently consider whether it is important and to the patient's best interest to perform surgery on the contralateral side on this day given the new intraoperative information of ipsilateral paralysis (3). According to Goretzki et al. (25), a failed IONM stimulation of RLN after resection of the first thyroid lobe is specific enough to reconsider the surgical strategy in patients with bilateral thyroid disease to prevent bilateral RLN palsy (25). Indeed, in $85 \%$ of cases with known nerve injury and in 56\% with negative IONM stimulation at the first side of dissection, the surgical strategy was changed with no postoperative bilateral RLN palsy. This was in contrast to the $17 \%$ bilateral RLN palsy $(\mathrm{P}<0.05)$ when surgeons were not aware of a preexisting or highly likely nerve injury at the first side of thyroid dissection (25).

After LOS on the first side of resection in bilateral goiter, more than $93 \%$ of surgeons declared their willingness to change the resection plan for the contralateral side to avoid the risk of bilateral RLN palsy. This resulted in a discontinuation of surgery (84\%) or in undertaking a less extensive resection for completion of the other side than originally planned (9\%) (7). Hospitals with the heaviest institutional caseload reportedly changed their surgical plans more often than those with a lower institutional caseload (7). Likewise, hospitals that routinely employed vagal stimulation and recording of EMG responses refrained from continuing the resection on the unaffected side more frequently than those that did not (7).

IONM is mentioned in the preoperative informed consent for primary and secondary interventions. If the patients are informed about the use of IONM, they should also be informed about the intraoperative consequences such as LOS. The possibility to stage the procedure in case of LOS on the first side must be specified in the preoperative informed consent. Patients are informed of the possibility of staging the contralateral side in case of LOS, in respect of the patient autonomy and with emphasis on shared decision making. In 
fact, most patients perfectly agree on this concept and comprehend the tremendous benefit in deciding whether to go to the other side in case of LOS at the first dominant side.

In a malpractice analysis, IONM provides important prognostic information regarding the ipsilateral VC function at the completion of the initial side of the surgery. IONM allows the surgeon to stage contralateral surgery if RLN damage is diagnosed, thereby avoiding the potential for bilateral VCP. If IONM is used, the surgeon has to consider the result and eventually stop the operation at the first side. If not otherwise stated, the operation has to be stopped in case of benign diseases. If the surgeon proceeds, he or she should outline the reasons (e.g., malignant bilateral disease, severe patient co-morbidity).

\section{IONM IN DIFFICULT THYROID PROCEDURES}

If an injury occurs, the surgeon must carefully examine arguments and justify the fact that monitoring was not used selectively in a difficult case. In 2009, we tested the hypothesis that the RLN identification during thyroid surgery reduces injury, and that intraoperative nerve monitoring may be of additional benefit (5). This represented the only randomized prospective trial for IONM vs. no-IONM. One thousand consenting patients scheduled to have bilateral thyroid surgery were randomized to standard protection or additional nerve monitoring. The primary outcome measure was the prevalence of RLN injury. Of 1,000 NAR in each group, transient and permanent RLN injuries were found respectively in 38 and 12 nerves without RLN monitoring ( $\mathrm{P}=0.011)$, and 19 and 8 nerves with RLN monitoring $(\mathrm{P}=0.368)$. The prevalence of transient RLN paresis was lowered by $2.9 \%$ in high-risk patients who had RLN monitoring $(\mathrm{P}=0.011)$ and $0.9 \%$ in low-risk patients $(\mathrm{P}=0.249)$. The negative and positive predictive values of RLN monitoring in predicting postoperative VC function were $98.9 \%$ and $37.8 \%$, respectively. We concluded that nerve monitoring decreased the incidence of transient, but not permanent, RLN paresis compared with visualization alone, particularly in high-risk patients (5).

The national thyroid surgeons' guidelines for IONM use propose IONM in risk operations (26). Thus, according to the above prospective trial and societies guidelines, thyroid surgeons should carefully consider justification if IONM was not used in high risk procedures $(5,26)$.

\section{IONM IN MALPRACTICE CLAIMS}

A recent analysis of claims of the years 1995 to 2014 ( $n=102)$ revealed a different pattern of published operational statistics due to the subjective and objective impairments of the quality of life (27). While the rate of persistent hypocalcemia and vocal palsy was low $(<3 \%)$ in surgical statistics, these complications dominated the causes of complaints in courts (unilateral vocal palsy, 30\%; bilateral vocal palsy, 20\%; hypoparathyroidism, 15\%). Unilateral and bilateral RLN paresis occurred during the first assessment period (1995-2000) predominantly after thyroid gland partial resections, and in the third observation period (2008-2014) after total thyroidectomy (27).

IONM has been used for the RLN functional control since 2001, and in 2008, in cases of vocal palsy, the dispute between plaintiffs and defendants at conciliation centers and courts 
has been the subject of almost all cases (27). In 80\% (2001-2007) and 70\% (2008-2014) of the RLN damage cases using the IONM, the specific use of neuromonitoring did not follow the recommendations published in the literature at the time of the damage $(3,27)$. The necessary standardized stimulation of the VN (V1 and V2) was dispensed, and only the signal tone after nerve stimulation was considered. Type 1 signal failures could not be detected in the absence of vagal stimulation, or artifact signals were confounded with regular muscle action potentials if the EMG were not considered (27).

Considering the fact that most vocal palsy is the result of nonstructural and exclusively functional RLN lesions, which are equally effective in vocal folds, it is understandable that bilateral vocal palsy occurred predominantly after thyroid operations without neuromonitoring. However, there is cause for concern that almost all bilateral vocal palsy have occurred during the last reported period (2008-2014) despite the use of neuromonitoring, mostly as a result of non-compliance with the standards (27).

We stated that, for the evaluation of a VCP after a thyroid surgery using IONM, it is of crucial importance to determine whether or not the standards for proper IONM use were applied correctly, and whether or not changes of neuromonitoring signal and EMG were correctly interpreted intraoperatively $(3,4,27)$. This results in the need for continuous surgical training in the correct application of standardized procedures of neuromonitoring, especially with regard to the indispensable consideration of EMG and vagal stimulation before and after resection of the first page in all planned bilateral resections.

\section{STANDARD OF CARE IN THYROID SURGERY}

The basis of a malpractice litigation (especially in the US) is the breach of the standard of care, i.e., if a surgeon provided a patient with care that is less than the standard and the patient was harmed, the surgeon will likely lose the lawsuit (28). The minimum standard of care is what other doctors in the area would provide. The questions remain as to: 1 ) what constitutes the standard of RLN management in the modern era of thyroid surgery; 2) what is the prevalence of neuromonitoring in thyroid surgery; and 3) how many surgeons use RLN monitoring. Horne et al. (29) reported in 2007 that more than $45 \%$ of the otolaryngologists use RLN monitoring. Sturgeon et al. (30) reported in 2009 that $37 \%$ of the general surgeons attending the American Association of Endocrine Surgeon meeting in 2006 used RLN monitoring; young surgeons (age, 35-55 years) and academic surgeons with high volume cases (probably dealing with more difficult cases) were most likely to use IONM always (30). This percentage increased from $7 \%$ to 37\% between 2001 to 2007 (31). In Germany, more than 50\% of thyroidectomies are performed with IONM (32). In Northern Europe, about 77\% of thyroidectomies are monitored (32). Thus, IONM will be the standard of care for thyroidectomy in a very near future.

\section{COST OF IONM}

In Italy, hospitalization costs for a thyroidectomy with IONM range from $€ 3,713$ to $€ 3,770$, that is $5 \%-7 \%$ higher than those for traditional thyroidectomy without IONM (33). Direct IONM cost ranged between $€ 250$ and $€ 270$ per single procedure (33). From personal communications, IONM costs are extremely different between countries, with even higher costs of $€ 800$ per procedure. There are patients who cannot afford the costs of such technology. Moreover, there 
are countries and health care systems that cannot afford such expenses. For this reason, the abovementioned issues should be carefully considered in these contexts.

\section{CONCLUSION}

In conclusion, we believe that the clinical benefits of IONM are definitely more important than the medico-legal issues presented here. Clinical benefits of IONM are both for the patients and the thyroid surgeons and include: 1) reduction of RLN injury in high risk procedures; 2) reduction of bilateral VC injuries; 3) reduction of permanent nerve injuries (34); 4) clear benefits for residents in training and less-experienced surgeons (6); 5) intraoperative, but not postoperative, evidence of RLN injury; 6) identification and dissection of the RLN; 7) facilitating the completeness of a total thyroidectomy; 8) serving the endoscopic thyroidectomy; and 9) providing an important instrument for research (35).

In case of a RLN palsy, the correct evaluation of all issues of the single and unique thyroid operation should be considered as: 1) the individual case (i.e., thyroid disease); 2) the procedure (i.e., central neck dissection); 3) the position of the surgeon (i.e., experience); 4) the experience of the surgeon with IONM; and 5) the documentation (printed|traceable। digital) of the VN stimulation. It is important in a monitored procedure, in the operative notes a statement that demonstrates that the RLN was visualized and was intact (IONM) at the end of the procedure. All these issues, together, are useful to draw conclusions from the standpoint of surgery, both for the surgical deliberation and for forensic.

The patient should be informed preoperatively not only of whether neuromonitoring is to be used during the imminent operation but also about the limitations of this technique and the surgical consequences resulting from a signal failure. This mainly includes the question of the strategy change of a planned bilateral operation if there has been a signal failure on the first operated side. Pre-operative RLN paresis should be clarified preoperatively and the patient should be informed of whether the resection of the opposite side can be spared, even if the benign struma is the larger, but not malignant, side. If there is an indispensable indication for the thyroid resection of the vocal fold palsy side, high-threshold prerequisites are attached to the surgeon's expertise.

IONM, comparably to the intraoperative parathormone determination established for decades, has shifted the previously postoperatively possible diagnosis into the operating room. Therefore, the use of neuromonitoring makes sense only if operative consequences are observed in the case of a recognizable loss of function. There are also marginal cases, which the surgeon "in dubio" should choose for the patient's safer procedure. For neuromonitoring, this means that, in the event of a signal failure of the $\mathrm{VN}$ on the first side, the resection of the second side should be avoided in order to prevent bilateral paresis.

Further studies should be carried out to determine whether a postoperatively non-injured vocal function can always be interpreted in cases where continuous neuromonitoring after signal failure indicates a recovery of the stimulation amplitude above $50 \%$ of the initial amplitude. For intraoperative management, it would be important to determine, without forcing any nerve-compromising actions (e.g., tension or compression of the nerve), how long after signal failure the amplitude should be maintained and whether, for example, intraoperative corticosteroids should be given. 


\section{REFERENCES}

1. Singer MC, Iverson KC, Terris DJ. Thyroidectomy-related malpractice claims. Otolaryngol Head Neck Surg 2012;146:358-61. PUBMED | CROSSREF

2. Abadin SS, Kaplan EL, Angelos P. Malpractice litigation after thyroid surgery: the role of recurrent laryngeal nerve injuries, 1989-2009. Surgery 2010;148:718-22. PUBMED | CROSSREF

3. Randolph GW, Dralle H, International Intraoperative Monitoring Study Group, Abdullah H, Barczynski $\mathrm{M}$, Bellantone R, et al. Electrophysiologic recurrent laryngeal nerve monitoring during thyroid and parathyroid surgery: international standards guideline statement. Laryngoscope 2011;121 Suppl 1:S1-16. PUBMED | CROSSREF

4. Tomoda C, Hirokawa Y, Uruno T, Takamura Y, Ito Y, Miya A, et al. Sensitivity and specificity of intraoperative recurrent laryngeal nerve stimulation test for predicting vocal cord palsy after thyroid surgery. World J Surg 2006;30:1230-3. PUBMED | CROSSREF

5. Barczyński M, Konturek A, Cichoń S. Randomized clinical trial of visualization versus neuromonitoring of recurrent laryngeal nerves during thyroidectomy. Br J Surg 2009;96:240-6. PUBMED | CROSSREF

6. Dralle H, Sekulla C, Lorenz K, Brauckhoff M, Machens AGerman IONM Study Group. Intraoperative monitoring of the recurrent laryngeal nerve in thyroid surgery. World J Surg 2008;32:1358-66. PUBMED | CROSSREF

7. Dralle H, Sekulla C, Lorenz K, Nguyen Thanh P, Schneider R, Machens A. Loss of the nerve monitoring signal during bilateral thyroid surgery. Br J Surg 2012;99:1089-95. PUBMED | CROSSREF

8. Godballe C, Madsen AR, Sørensen CH, Schytte S, Trolle W, Helweg-Larsen J, et al. Risk factors for recurrent nerve palsy after thyroid surgery: a national study of patients treated at Danish departments of ENT Head and Neck Surgery. Eur Arch Otorhinolaryngol 2014;271:2267-76. PUBMED | CROSSREF

9. Adam MA, Thomas S, Youngwirth L, Hyslop T, Reed SD, Scheri RP, et al. Is there a minimum number of thyroidectomies a surgeon should perform to optimize patient outcomes? Ann Surg 2017;265:402-7. PUBMED | CROSSREF

10. Lorenz K, Abuazab M, Sekulla C, Schneider R, Nguyen Thanh P, Dralle H. Results of intraoperative neuromonitoring in thyroid surgery and preoperative vocal cord paralysis. World J Surg 2014;38:582-91. PUBMED | CROSSREF

11. Lorenz K, Sekulla C, Schelle J, Schmeiss B, Brauckhoff M, Dralle HGerman Neuromonitoring Study Group. What are normal quantitative parameters of intraoperative neuromonitoring (IONM) in thyroid surgery? Langenbecks Arch Surg 2010;395:901-9. PUBMED | CROSSREF

12. Brauckhoff M, Machens A, Sekulla C, Lorenz K, Dralle H. Latencies shorter than $3.5 \mathrm{~ms}$ after vagus nerve stimulation signify a nonrecurrent inferior laryngeal nerve before dissection. Ann Surg 2011;253:1172-7. PUBMED | CROSSREF

13. Chiang FY, Lee KW, Chen HC, Chen HY, Lu IC, Kuo WR, et al. Standardization of intraoperative neuromonitoring of recurrent laryngeal nerve in thyroid operation. World J Surg 2010;34:223-9. PUBMED | CROSSREF

14. Chiang FY, Lu IC, Kuo WR, Lee KW, Chang NC, Wu CW. The mechanism of recurrent laryngeal nerve injury during thyroid surgery--the application of intraoperative neuromonitoring. Surgery 2008;143:743-9. PUBMED | CROSSREF

15. Hamelmann WH, Meyer T, Timm S, Timmermann W. A critical estimation of intraoperative neuromonitoring (IONM) in thyroid surgery. Zentralbl Chir 2002;127:409-13. PUBMED | CROSSREF

16. Thomusch O, Sekulla C, Machens A, Neumann HJ, Timmermann W, Dralle H. Validity of intra-operative neuromonitoring signals in thyroid surgery. Langenbecks Arch Surg 2004;389:499-503. PUBMED | CROSSREF

17. Beldi G, Kinsbergen T, Schlumpf R. Evaluation of intraoperative recurrent nerve monitoring in thyroid surgery. World J Surg 2004;28:589-91. PUBMED | CROSSREF 
18. Hermann M, Hellebart C, Freissmuth M. Neuromonitoring in thyroid surgery: prospective evaluation of intraoperative electrophysiological responses for the prediction of recurrent laryngeal nerve injury. Ann Surg 2004;240:9-17. PUBMED | CROSSREF

19. Chan WF, Lang BH, Lo CY. The role of intraoperative neuromonitoring of recurrent laryngeal nerve during thyroidectomy: a comparative study on 1000 nerves at risk. Surgery 2006;140:866-72. PUBMED | CROSSREF

20. Wong KP, Mak KL, Wong CK, Lang BH. Systematic review and meta-analysis on intra-operative neuromonitoring in high-risk thyroidectomy. Int J Surg 2017;38:21-30. PUBMED | CROSSREF

21. Melin M, Schwarz K, Pearson MD, Lammers BJ, Goretzki PE. Postoperative vocal cord dysfunction despite normal intraoperative neuromonitoring: an unexpected complication with the risk of bilateral palsy. World J Surg 2014;38:2597-602. PUBMED | CROSSREF

22. Schneider R, Randolph GW, Sekulla C, Phelan E, Thanh PN, Bucher M, et al. Continuous intraoperative vagus nerve stimulation for identification of imminent recurrent laryngeal nerve injury. Head Neck 2013;35:1591-8. PUBMED | CROSSREF

23. Dionigi G, Chiang FY, Dralle H, Boni L, Rausei S, Rovera F, et al. Safety of neural monitoring in thyroid surgery. Int J Surg 2013;11 Suppl 1:S120-6.

PUBMED | CROSSREF

24. Echternach M, Maurer CA, Mencke T, Schilling M, Verse T, Richter B. Laryngeal complications after thyroidectomy: is it always the surgeon? Arch Surg 2009;144:149-53.

PUBMED | CROSSREF

25. Goretzki PE, Schwarz K, Brinkmann J, Wirowski D, Lammers BJ. The impact of intraoperative neuromonitoring (IONM) on surgical strategy in bilateral thyroid diseases: is it worth the effort? World J Surg 2010;34:1274-84. PUBMED | CROSSREF

26. Dralle H, Lorenz K, Schabram P, Musholt TJ, Dotzenrath C, Goretzki PE, et al. Intraoperative neuromonitoring in thyroid surgery. Recommendations of the Surgical Working Group for Endocrinology. Chirurg 2013;84:1049-56. PUBMED | CROSSREF

27. Dralle H, Schneider R, Lorenz K, Phuong NT, Sekulla C, Machens A. Vocal cord paralysis after thyroid surgery: current medicolegal aspects of intraoperative neuromonitoring. Chirurg 2015;86:698-706. PUBMED | CROSSREF

28. Angelos P. Recurrent laryngeal nerve monitoring: state of the art, ethical and legal issues. Surg Clin North Am 2009;89:1157-69.

PUBMED | CROSSREF

29. Horne SK, Gal TJ, Brennan JA. Prevalence and patterns of intraoperative nerve monitoring for thyroidectomy. Otolaryngol Head Neck Surg 2007;136:952-6. PUBMED | CROSSREF

30. Sturgeon C, Sturgeon T, Angelos P. Neuromonitoring in thyroid surgery: attitudes, usage patterns, and predictors of use among endocrine surgeons. World J Surg 2009;33:417-25. PUBMED | CROSSREF

31. Timmermann W, Hamelmann WH, Thomusch O, Sekulla C, Grond S, Neumann HJ, et al. Effectiveness and results of intraoperative neuromonitoring in thyroid surgery. Statement of the Interdisciplinary Study Group on Intraoperative Neuromonitoring of Thyroid Surgery. Chirurg 2004;75:916-22. PUBMED | CROSSREF

32. Godballe C. Registry of surgical results: organization and outcomes. 34th Annual Meeting of the European Thyroid Association; 2009 Sep 5-9; Lisbon, Portugal. Altdorf: European Thyroid Association; 2009.

33. Dionigi G, Bacuzzi A, Boni L, Rausei S, Rovera F, Dionigi R. Visualization versus neuromonitoring of recurrent laryngeal nerves during thyroidectomy: what about the costs? World J Surg 2012;36:748-54. PUBMED | CROSSREF

34. Schneider R, Sekulla C, Machens A, Lorenz K, Nguyen Thanh P, Dralle H. Postoperative vocal fold palsy in patients undergoing thyroid surgery with continuous or intermittent nerve monitoring. Br J Surg 2015;102:1380-7. PUBMED | CROSSREF

35. Lin YC, Dionigi G, Randolph GW, Lu IC, Chang PY, Tsai SY, et al. Electrophysiologic monitoring correlates of recurrent laryngeal nerve heat thermal injury in a porcine model. Laryngoscope 2015;125:E283-90. PUBMED | CROSSREF 